Research article

Open Access

\title{
Intervertebral disc cells as competent phagocytes in vitro: implications for cell death in disc degeneration
}

\author{
Philip Jones ${ }^{1,2}$, Lucy Gardner ${ }^{1,2}$, Janis Menage ${ }^{1}$, Gwyn T Williams ${ }^{2}$ and Sally Roberts ${ }^{1,2}$
}

\author{
${ }^{1}$ Centre for Spinal Studies, Robert Jones \& Agnes Hunt Orthopaedic \& District Hospital NHS Trust, Oswestry, Shropshire SY10 7AG, UK \\ 2Institute of Science and Technology in Medicine, Keele University, Keele, Staffordshire, ST5 5BG, UK \\ Corresponding author: Sally Roberts, sally.roberts@rjah.nhs.uk
}

Received: 8 Apr 2008 Revisions requested: 23 May 2008 Revisions received: 19 Jun 2008 Accepted: 1 Aug 2008 Published: 1 Aug 2008

Arthritis Research \& Therapy 2008, 10:R86 (doi:10.1186/ar2466)

This article is online at: http://arthritis-research.com/content/10/4/R86

(c) 2008 Jones et al.; licensee BioMed Central Ltd.

This is an open access article distributed under the terms of the Creative Commons Attribution License (http://creativecommons.org/licenses/by/2.0), which permits unrestricted use, distribution, and reproduction in any medium, provided the original work is properly cited.

\begin{abstract}
Introduction Apoptosis has been reported to occur in the intervertebral disc. Elsewhere in the body, apoptotic cells are cleared from the system via phagocytosis by committed phagocytes such as macrophages, reducing the chance of subsequent inflammation. These cells, however, are not normally present in the disc. We investigated whether disc cells themselves can be induced to become phagocytic and so have the ability to ingest and remove apoptotic disc cells, minimising the damage to their environment.
\end{abstract}

Method Bovine nucleus pulposus cells from caudal intervertebral discs were grown in culture and exposed to both latex particles (which are ingested by committed phagocytes) and apoptotic cells. Their response was monitored via microscopy, including both fluorescent and video microscopy, and compared with that seen by cell lines of monocytes/ macrophages (THP-1 and J774 cells), considered to be committed phagocytes, in addition to a nonmacrophage cell line (L929 fibroblasts). Immunostaining for the monocyte/ macrophage marker, CD68, was also carried out.
Results Disc cells were able to ingest latex beads at least as efficiently, if not more so, than phagocytic THP-1 and J774 cells. Disc cells ingested a greater number of beads per cell than the committed phagocytes in a similar time scale. In addition, disc cells were able to ingest apoptotic cells when cocultured in monolayer with a UV-treated population of HeLa cells. Apoptotic disc cells, in turn, were able to stimulate phagocytosis by the committed macrophages. CD68 immunostaining was strong for THP-1 cells but negligible for disc cells, even those that had ingested beads.

Conclusion In this study, we have shown that intervertebral disc cells are capable of behaving as competent phagocytes (that is, ingesting latex beads) and apoptotic cells. In terms of number of particles, they ingest more than the monocyte/macrophage cells, possibly due to their greater size. The fact that disc cells clearly can undergo phagocytosis has implications for the intervertebral disc in vivo. Here, where cell death is reported to be common yet there is normally no easy access to a macrophage population, the endogenous disc cells may be encouraged to undergo phagocytosis (for example, of neighbouring cells within cell clusters).

\section{Introduction}

Cells are the vital machinery for synthesising and maintaining the functioning matrix in all tissues and the intervertebral disc within the spine is no different. Cell death within the disc cell population has been reported to be a common phenomenon and recently there have been several studies showing that apoptosis, or controlled cell death, occurs here [1-7]. Apoptosis is a genetically controlled mechanism that is considered to be important for tissue homeostasis. The cell dies in a welldefined process involving condensation of the chromatin and packaging of cell components within lipid membranes to form apoptotic bodies, thus minimising any subsequent damage to the surrounding matrix $[8,9]$. This is in contrast to necrosis, which is relatively uncontrolled with the cell membrane disrupting and releasing cellular contents. Necrosis is believed to be more damaging to the tissue with the release of degradative enzymes and the ability to illicit an inflammatory response [10].

Apoptosis is often described as a 'silent death' [11] with cells being destroyed from within [12] and the remains of the cell subsequently 'eaten' by phagocytic cells, effectively eliminating all physical evidence of death. In most tissues, this 
clearance of apoptotic cells will be undertaken by the committed phagocytes of the macrophage lineage, available via the local blood supply. However, the normal adult intervertebral disc has little or no direct vasculature supplying it, particularly the central nucleus pulposus (NP) [13], where cell death is reported to be most common [14]. This raises the question of how apoptotic cells within the intervertebral disc might be cleared. Other cell types have been reported to be induced to phagocytose when exposed to stimuli if macrophages are not available (for example, epithelial, endothelial, and tumour cells) [15]. The mechanism is not fully understood, but dying cells appear to elicit 'eat me' signals (for example, exposure of a phosphatidylserine molecule on the outer surface of the cell membrane [16] which can stimulate other cells to become phagocytic, albeit as facultative phagocytes).

We hypothesised that intervertebral disc cells could behave in this manner and that, if exposed to appropriate stimuli such as apoptotic cells, they could be induced to become phagocytic. This in vitro study, comparing the response of bovine NP cells with that of committed phagocytes to exposure both to latex beads (a commonly used stimulus for phagocytosis) and to apoptotic cells, has demonstrated this to be the case.

\section{Materials and methods Nucleus pulposus cell extraction and cell lines}

NP was dissected from the centre of the three uppermost bovine caudal discs obtained from young adult cattle $(n=15$, ages 18 to 32 months) within 1 hour of death with permission from a local abattoir. The tissue of the three discs was pooled and the NP cells were isolated by incubating the diced tissue overnight at $37^{\circ} \mathrm{C}$ in $0.8 \mathrm{mg} / \mathrm{mL}$ crude type $\mathrm{XI}$ collagenase (Sigma-Aldrich, Gillingham, Dorset, UK) containing 1.67 units per millilitre DNase (Sigma-Aldrich). The cells obtained after digestion were washed using Dulbecco's modified Eagle's medium (DMEM)/F-12 (Invitrogen Corporation, Paisley, UK) supplemented with 10\% foetal bovine serum (FBS) (PAA Laboratories, Yeovil, Somerset, UK) and were centrifuged at 107 $g$ for 10 minutes. The cells were then filtered through a $70-\mu \mathrm{m}$ nylon cell strainer (BD Biosciences, Cowley, UK). The extracted cells were grown in monolayer culture in DMEM/F12 in a fully humidified atmosphere with $5 \% \mathrm{CO}_{2}$ and $21 \% \mathrm{O}_{2}$ at $37^{\circ} \mathrm{C}$. The cells were expanded and passaged twice before use.

Two cell lines, THP-1 and J774 cells, were used as committed phagocytes whilst the fibroblast cell line, L929, was used for comparison as a nonmacrophage-like cell and HeLa was used to provide a source of apoptotic cells. The human monocytic leukaemic cell line, THP-1 [17] (European Collection of Cell Cultures [ECACC], Salisbury, UK), was maintained in RPMI 1640 medium (Invitrogen Corporation) supplemented with 10\% FBS. The $\mathbf{J 7 7 4}$ cell line (derived from murine macrophages, kindly donated by Robin May, University of Birmingham, UK) was maintained in DMEM/F-12 supplemented with
$10 \%$ FBS. HeLa cells (donated by Mann Nguyen, Robert Jones \& Agnes Hunt Orthopaedic \& District Hospital NHS Trust, Oswestry, Shropshire, UK), an immortalised epithelial cell line, were also maintained in RPMI 1640 supplemented with 10\% FBS. L929, a mouse fibroblast cell line, was obtained from ECACC (number 85011425) and cells were grown in DMEM/F-12 and 10\% FBS. This study does not involve human subjects, human tissue, or experimentation on animals.

\section{Phagocytosis assays using latex beads}

To activate the THP-1 to a macrophage phenotype, the cells were treated with $160 \mathrm{nM}$ phorbol 12-myristrate 13-acetate (PMA) (Sigma-Aldrich) for 72 hours [18]. The cells were washed with RPMI and then incubated with RPMI containing $0.02 \% 2-\mu \mathrm{m}$-diameter fluorescein isothiocyanate (FITC)-latex beads (Sigma-Aldrich). Alternatively, to activate the J774 cells to a macrophage phenotype, they were treated with $243 \mathrm{nM}$ PMA for 2 hours, washed with DMEM/F-12, and then incubated with DMEM/F-12 containing 0.02\% 2- $\mu$ m-diameter FITC-latex beads [19]. NP cells were grown in monolayer culture for 24 hours before adding latex beads as above. Cells were grown at a concentration of 500,000 cells per well in sixwell plates. At time points between 0 and 48 hours (approximately $1,2,4,6,8,24,32$, and 48 hours), cultures were fixed in methanol for 5 minutes, before staining with Jenner-Giemsa stain [20]. Two hundred cells for each culture were observed and the number counted which had ingested any latex beads in addition to the number of cells (a) that had not ingested any beads and that had ingested (b) 1 to 4 beads, (c) 5 to 10 beads, and (d) more than 10 beads (Figure 1). All cultures for each set of measurements were done in quadruplicate.

\section{Phagocytosis of apoptotic HeLa and nucleus pulposus cells by THP-1 and $\mathbf{J 7 7 4}$ cells}

Both THP-1 and J774 cells were fluorescently tagged using a green fluorescent cell linker mini kit (catalogue number MINI67; Sigma-Aldrich) [21] and then pretreated with PMA as described above. Cells to be induced to become apoptotic, whether NP or HeLa cells, were fluorescently tagged using a red fluorescent cell linker mini kit (catalogue number MINI-26; Sigma-Aldrich). NP or HeLa cells $\left(1 \times 10^{6}\right)$ in $2 \mathrm{~mL} /$ well were tagged. Apoptosis was induced in the NP and HeLa cells by exposing them to UV light (UVG54 grid lamp; UVP Ltd, Cambridge, Cambridgeshire, UK) at a distance of $15 \mathrm{~cm}$ for $3 \mathrm{~min}$ utes [22], providing a UV dose of approximately $560 \mathrm{~W} / \mathrm{cm}^{2}$. This has been shown to cause approximately $50 \%$ of the cells to become apoptotic (as can be seen with Hoechst 33342 dye, Figure 2). These cells were then added to the activated THP-1 or J774 cells at a ratio of 2 UV-treated cells to 1 normal cell. Time-lapse video microscopy using a digital video camera (TK-1280E; JVC, Yokohama, Japan) was used to capture images every 5 minutes over the span of 72 hours. The digital images were then converted into video files using Media Studio Video Editor (version 3.5; Ulead System Inc., Karst, 
Figure 1

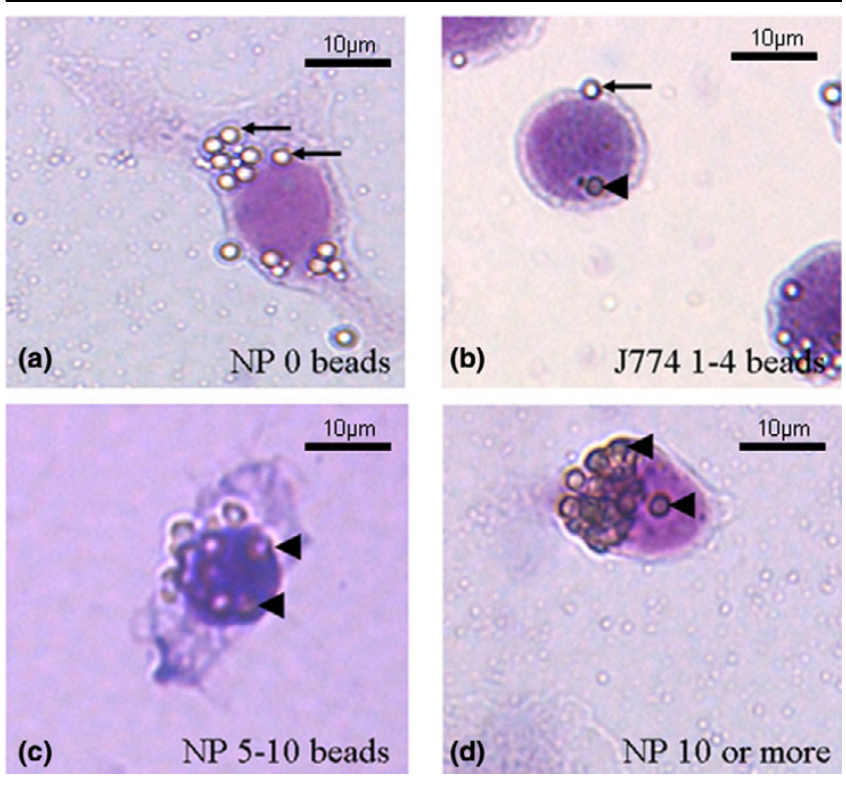

Phagocytosis of latex beads by intervertebral disc and other cell types. Bovine nucleus pulposus (NP) cells or $\mathrm{J774}$ cells that ingested (a) 0 latex beads, (b) 1 to 4 beads, (c) 5 to 10 beads, and (d) more than 10 beads are shown (Jenner-Giemsa stain). Beads outside the cell membrane are clearly more birefringent and brighter (arrows) than those ingested, which have a dull appearance (arrowhead).

Germany). Cells were also observed by means of a fluorescent microscope (Leica DMBL; Leica Microsystems GmbH, Wetzlar, Germany) after 24, 48, and 72 hours. Images were taken

Figure 2

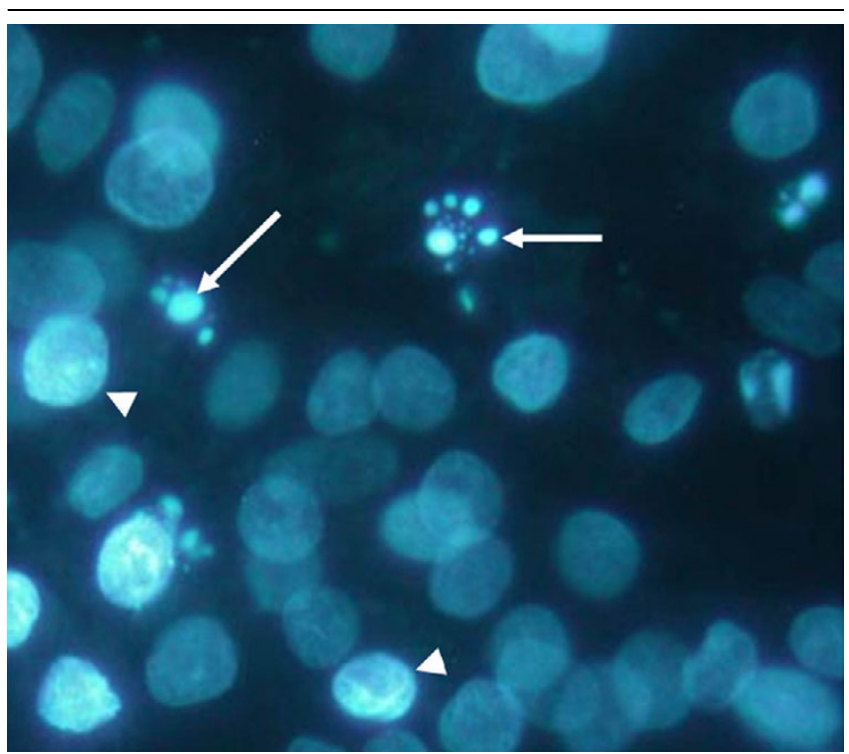

Morphology of apoptotic disc cells. Hoechst 33342-stained nucleus pulposus cells demonstrate typical apoptotic morphology after 3 minutes of UV treatment: condensation of the nuclear material (arrowheads) followed by formation of apoptotic bodies (arrows). Original magnification: $\times 630$. using IPLab Scientific Imaging Software (Scanalytics Inc., part of BD Biosciences).

\section{Phagocytosis of apoptotic cells by nucleus pulposus cells}

NP cells were fluorescently tagged red with a cell linker kit (Sigma-Aldrich) and allowed to adhere overnight. NP cells (1 $\left.\times 10^{5}\right)$ in $2 \mathrm{~mL} /$ well were used. HeLa cells $\left(5 \times 10^{5}\right.$ per well) were fluorescently tagged green and exposed to UV light for 3 minutes as above. They were then added to the NP cells and left for up to 72 hours. Time-lapse video microscopy and fluorescent images were taken at 24,48 , and 72 hours and used to observe the two populations within the cocultures, redlabelled NP cells in monolayer with the green-labelled HeLa cells (apoptotic).

\section{CD68 immunostaining}

Immunostaining for CD68 was carried out on bovine NP cells cultured both with and without latex beads for $0,4,6$, and 24 hours, in addition to THP-1 cells, pretreated with PMA, and grown on coverslips for 24 hours. Slides were fixed in acetone and incubated with SSC (150 mM sodium chloride and 15 $\mathrm{mM}$ sodium citrate at $55^{\circ} \mathrm{C}$ ). Endogenous peroxidase was blocked with $0.3 \%$ hydrogen peroxide in methanol and further blocking was performed by incubating the sections with normal serum. Sections were then incubated with an antibody to CD68 (1:23 in phosphate-buffered saline; Dako, Glostrup, Denmark, clone EBM11). Labelling was visualised with peroxidase and diaminobenzadine as the substrate and enhanced with avidin-biotin complex (Vector Laboratories, Burlingame, CA, USA). Mouse IgG was used as a negative control. Immunopositivity was assessed by recording at least 200 cells for each cell culture at each time point.

\section{Results \\ Response of committed phagocytes and fibroblasts to exposure to latex beads}

PMA-activated THP-1 and J774 cells responded to being cultured with latex beads, by ingesting significant numbers of them with time, as previously described [18]. Two hours after exposure, $27.8 \%$ and $16.4 \%$ of THP-1 and $\mathrm{J} 774$ cells, respectively, had ingested at least some beads, with maximum ingestion at around 6 hours for both cell types (Figure $3 a$ and $3 b$ ). Forty-nine percent of THP-1 cells and $38.6 \%$ of $\mathrm{J} 774$ cells had ingested beads at this time point, with $5.4 \%$ and $1.7 \%$, respectively, having ingested more than four beads per cell (Figures $4 \mathrm{a}$ and $4 \mathrm{~b}$ ). At time points beyond 6 hours, the number of both cell types with obvious beads ingested reduced. In contrast to the committed phagocytes and NP cells (see below), only $2.5 \%$ of L929 fibroblast cells had ingested any beads at 6 hours, and only $0.1 \%$ of them with more than four beads per cell. 
Figure 3

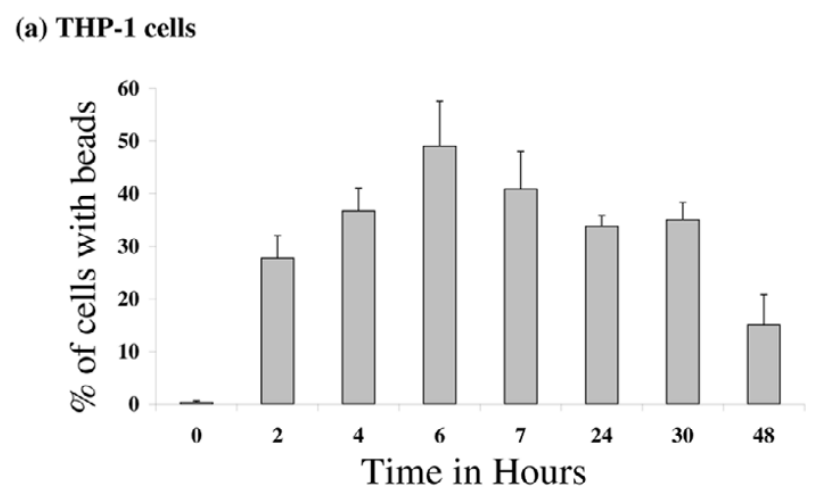

(b) J774 cells

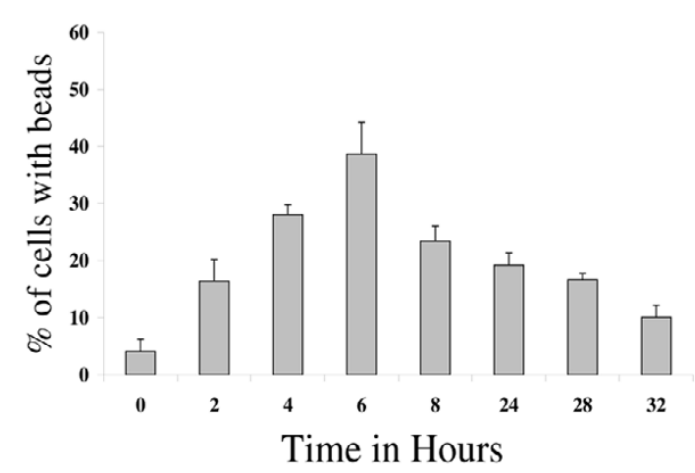

(c) Bovine nucleus cells

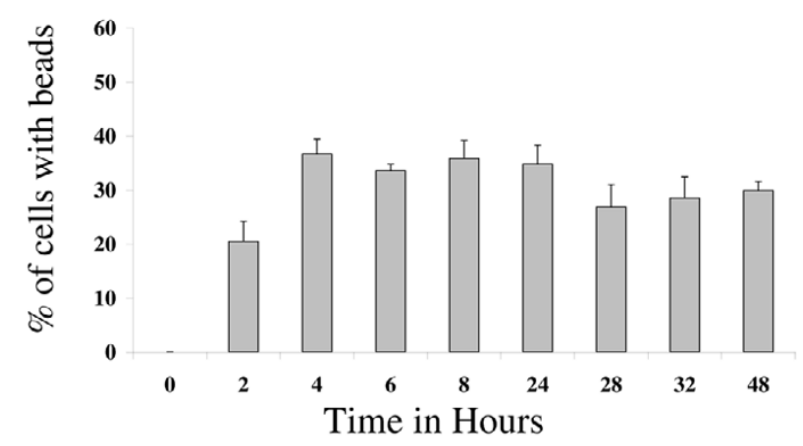

Frequency of beads ingested by cells. Bar charts present the percentage of cells that had ingested beads between 0 and 48 hours after the addition of latex beads for all three cell types investigated: (a) THP-1, (b) $\mathrm{J} 774$, and (c) nucleus pulposus cells. Bar indicates standard error ( $n=4$ cultures for each time point).

\section{Response of nucleus pulposus cells to exposure to latex beads}

NP cells also responded to being cultured with latex beads by ingesting them. After 2 hours, 20.5\% of cells had ingested some beads, but this increased to a maximum of $36.7 \%$ at 4 hours, after which the number decreased slightly with time, but not to levels as low as seen for THP-1 or J774 cells (Figure 3c). At 6 hours, $16.7 \%$ of NP cells had ingested more than four beads per cell (Figure 4c).
Figure 4
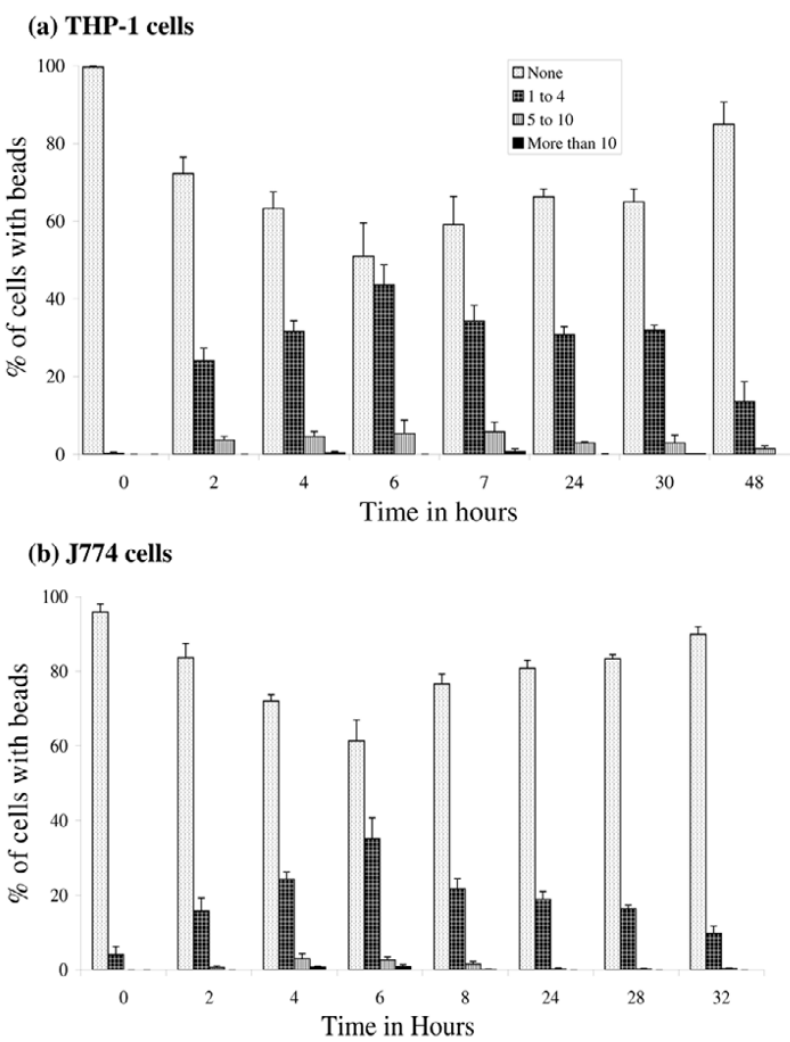

(c) Bovine nucleus pulposus cells

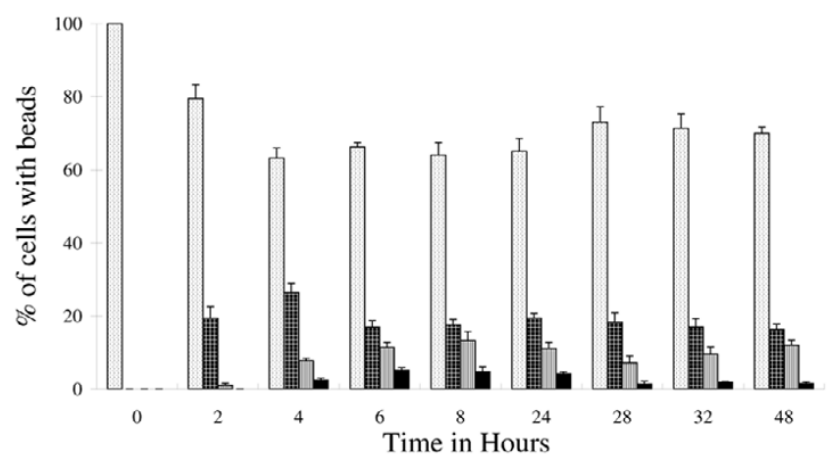

Numbers of beads ingested by cells. Frequency bar chart shows the percentage of cells that had ingested different numbers of latex beads with time: (a) THP-1 cells, (b) J774 cells, and (c) nucleus pulposus cells. Bar indicates standard error ( $n=4$ cultures for each time point).

\section{Phagocytosis of apoptotic cells by committed phagocytes and nucleus pulposus cells}

Coculturing of both the committed phagocytes, J774 and THP-1 cells, with HeLa or NP cells that had been UV-treated to render them apoptotic was monitored by video microscopy. It was possible, over a period of several hours, to follow certain J774 and THP-1 cells as they moved around the tissue culture flasks, made contact with an apoptotic cell or apoptotic body, and subsequently ingested it. Such a sequence demonstrating this activity with apoptotic disc cells has been separated 

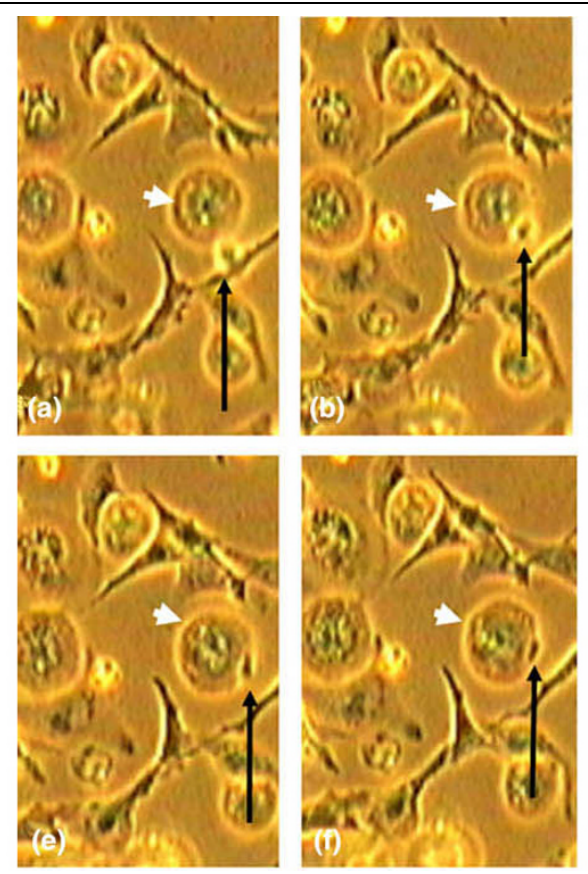
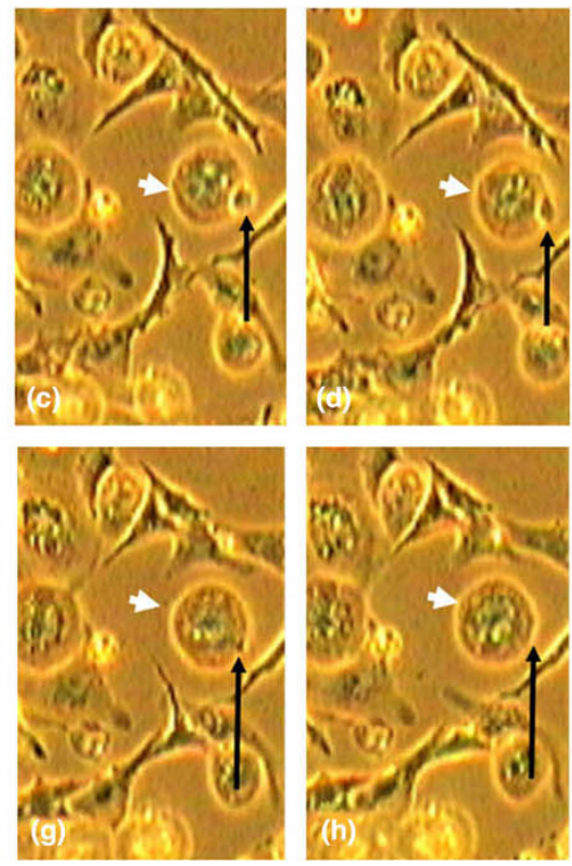

Apoptotic disc cells stimulating phagocytosis by other cells. A sequence of phase-contrast images obtained from video microscopy shows a THP-1 cell (white arrow) ingesting an apoptotic, phase-bright nucleus pulposus cell (black arrow). These frames demonstrate that apoptotic disc cells can trigger a phagocytic response. Images were taken over the course of 40 minutes. Original magnification $\times 100$.

into individual still shots and is shown in Figure 5, demonstrating that apoptotic disc cells can stimulate their phagocytosis by the committed macrophages. Similarly, NP cells cocultured with UV-treated apoptotic cells could be seen to phagocytose and ingest the apoptopic cells (Figure 6). This demonstrated that disc cells can be 'switched on' to phagocytose apoptopic cells (Figure 6).

\section{CD68 immunostaining}

Immunopositivity for CD68 was seen in none of the NP cells without exposure to latex beads and in very few cells exposed to beads $(<2 \%$ of cells). In contrast, greater than $95 \%$ of THP1 cells were immunopositive (Figure 7).

\section{Discussion}

Removal of apoptotic cells by phagocytes is considered to be the final common event in the life of most apoptotic cells [23]. Efficient clearance before lysis occurs is critical to tissue health and integrity. Phagocytic clearance of apoptotic cells by macrophages or facultative phagocytic cells constitutes an integral part of the overall suicide process [24] and is thought to provide a safe disposal route [25]. In addition, it can actively downregulate inflammatory responses.

\section{Figure 6}
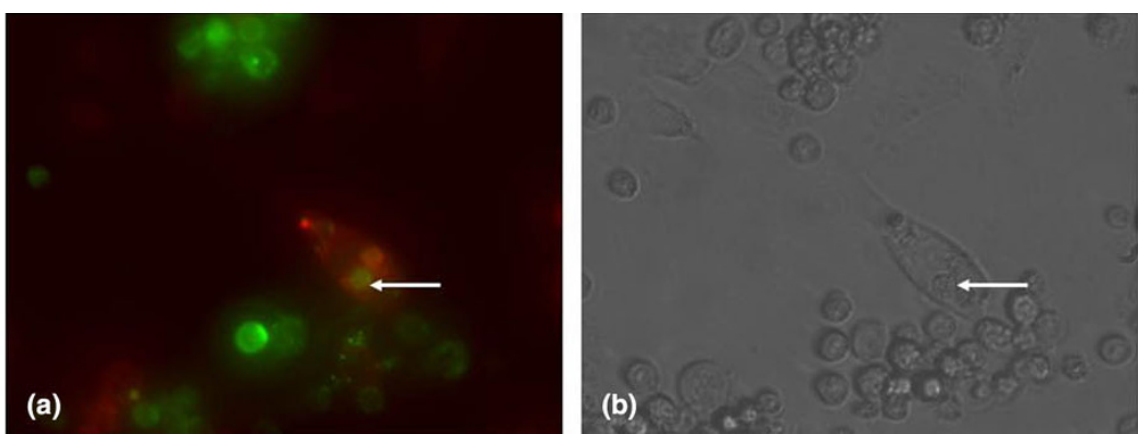

Intervertebral disc cells stimulated to undergo phagocytosis. Fluorescently tagged (red) bovine nucleus pulposus cells ingest fluorescently tagged (green) apoptotic HeLa cells: (a) fluorescent image and (b) phase-contrast image of the same field. This sequence demonstrates that disc cells are capable of phagocytosing apoptotic cells. Arrow shows ingested apoptotic body. Original magnification $\times 400$. 

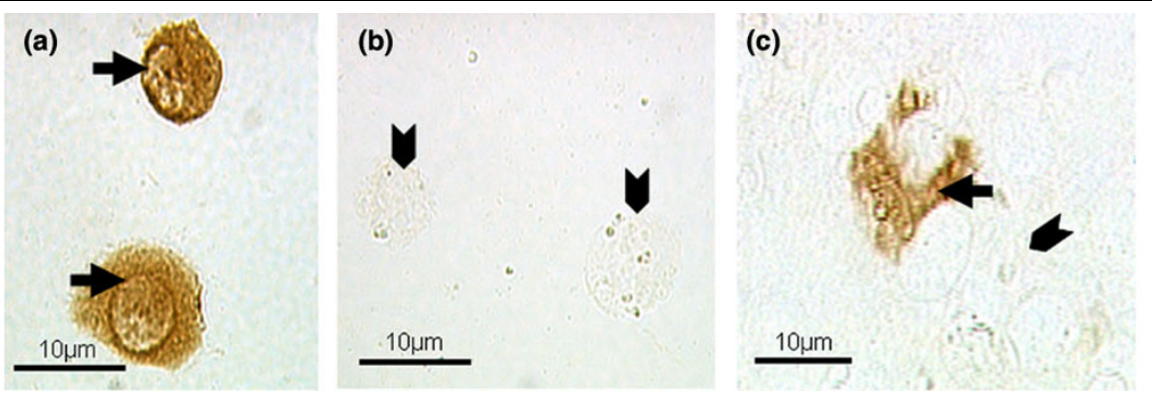

CD68 immunostaining of THP-1 and bovine nucleus pulposus cells. (a) Virtually all THP-1 cells were positively immunostained with the CD68 antibody (arrows) and (b) negatively immunostained with the normal mouse lgG antibody (arrowheads). (c) There were very few positively stained bovine nucleus pulposus cells for CD68 (arrow). Most were negative (arrowhead).

Whilst apoptosis of cells within the intervertebral disc has been investigated by several groups, little attention has been paid to the subsequent physiological process and clearance of the apoptotic cells. The frequency of apoptosis is unclear, with $50 \%$ to $70 \%$ of cells being reported as apoptotic by some workers [2] (or at least as being TUNEL [terminal deoxynucleotidyl transferase-mediated dUTP-biotin nick end-labeling]-positive), but thought to be considerably less by others [26]. Whatever the incidence, the presence of apoptotic cells would not cause concern if the disc were a vascularised tissue where macrophages could eliminate such cells from the environment. However, the adult healthy disc is considered to be the largest avascular tissue in the body [27], so the accessibility of macrophages is likely to be more limited than in most tissues. Other cell types, including fibroblasts, glomerular mesangial cells, endothelial cells, and even chondrocytes, have been reported to be capable of phagocytic ingestion of apoptotic cells of the same lineage in the absence of committed phagocytes $[15,16,28]$.

Molecules that may trigger engulfment by committed phagocytes have been identified to some extent (including phosphatidylserine, recognised by receptors such as CD36, CD68, or CD14), but much less is known about the molecular cascade that may occur with the 'amateur' or facultative phagocytes $[15,23]$. Lectins have been suggested to be more important in facultative phagocytes than the committed population [16]. It is suggested that phagocytosis of apoptotic cells, however it occurs, may be beneficial to the tissue in more ways than one. In addition to removing potential matrix-degrading enzymes, phagocytosis can trigger the release of antiinflammatory cytokines such as transforming growth factorbeta whilst inhibiting the production of proinflammatory cytokines, including tumour necrosis factor-alpha [11].

Whilst macrophages or mononuclear cells have been reported to occur in herniated extruded intervertebral discs [29], they are not observed in healthy discs. Markers typical of macrophages have been reported in cells within the intervertebral disc. Virri and colleagues [30] have described 55\% of herni- ated human discs to contain CD68-immunopositive cells, but only in areas close to blood vessels. Nerlich and colleagues [31] found no CD68-positive cells in discs of foetus, infants, or adolescents. In contrast, some CD68-positive cells were seen in the NP of all discs showing disc degeneration, but the morphology of these cells was no different from that of the cells normally found here. Thus, the authors suggest that the CD68-positive cells are not invaded monocytes or macrophages but transformed resident cells that are involved in phagocytosis. Our study would support this and provides evidence that NP disc cells can indeed behave as phagocytes and undertake phagocytosis, at least in vitro. Indeed, they are able to ingest more latex beads than the committed phagocytes, although this may be, in part, a reflection of their larger size (disc cells had a mean area of $544 \pm 135 \mu \mathrm{m}^{2}$ compared with $160 \pm 93 \mu \mathrm{m}^{2}$ for THP-1 cells and $175 \pm 49 \mu \mathrm{m}^{2}$ for $\mathrm{J} 774$ cells in monolayer). In addition, the disc cells appeared to be able to retain the beads that they had ingested better than the committed phagocytes, in which the number of beads internalised decreased with time, perhaps due to subsequent extrusion of some beads, as has been seen by macrophages previously [32]. Disc cells were certainly much more effective at ingesting the beads than the fibroblast cell line, L929. Cells in other cartilaginous tissue are also reported to be capable of becoming phagocytic; for example, articular cartilage chondrocytes have been shown to behave in a similar manner [28] and occasionally the cells in epiphyseal cartilage [33]. The lack of CD68 expression by the disc cells in this study may be a feature of in vitro culture as CD68 has been shown to be expressed by osteoblasts with increasing time in culture, independently of how many particles had been phagocytosed [34]. However, in the study on osteoblasts, CD68 was found after 72 hours in culture (with no earlier observations); in the current study, NP cells were cultured for a maximum of only 48 hours.

The capability of disc cells to phagocytose and the capability of apoptopic disc cells to stimulate phagocytosis could be beneficial to the intervertebral disc in vivo in many ways. Death of the cells within the intervertebral disc is reported to be extensive [35] and increasingly common after the age of 11 in 
humans [36]. Efficient clearance of the dying cells, whether they have died by apoptosis or autophagy, is advantageous. If they are not cleared, it may lead to secondary necrosis with its subsequent harmful impact on the cells' environment [24] and possible stimulation of an inflammatory response. It also provides a means of clearing senescent cells, of which there are plenty in the intervertebral disc, particularly in herniated discs [37] or degenerate discs [38,39]. Although disc cells often occur in isolation with much matrix around them, in degenerate discs, clusters of cells, with individual cells directly contacting their neighbours, are a common feature. Whilst migration of disc cells through the matrix may be an unlikely phenomenon, phagocytosis of neighbouring or adjacent cells in clusters would appear to be perfectly feasible.

\section{Conclusion}

In summary, this study shows that bovine NP cells are able of phagocytosing latex beads and apoptotic bodies. The pattern of phagocytosis of beads is comparable to the pattern shown by the committed phagocytes, THP1 and J774 cell lines. This, together with earlier reports that disc cells can express CD68, a phagocytic marker [31], suggests that the cells of the intervertebral disc may be capable of the removal of apoptotic bodies when disc cells die in vivo via apoptosis or indeed any variation such as autophagy [24]. This could have very important physiological implications since removal of apoptotic cells is likely to be the most important event in vivo if tissue structure and function are to be maintained in the face of major cell loss [23].

\section{Competing interests}

The authors declare that they have no competing interests.

\section{Authors' contributions}

PJ and LG carried out the practical laboratory work. JM assisted with data analysis and writing the manuscript. GTW provided expert advice. SR raised funds, conceived the research, and wrote the manuscript. All authors read and approved the final manuscript.

\section{Acknowledgements}

We are grateful to the Arthritis Research Campaign for financial support (grant 16143).

\section{References}

1. Lotz JC, Colliou OK, Chin JR, Duncan NA, Liebenburg E: Compression-induced degeneration of the intervertebral disc: an in vivo mouse model and finite-element study. Spine 1998, 23:2493-2506

2. Gruber HE, Hanley EN: Analysis of aging and degeneration of the human intervertebral disc: comparison of surgical specimens with normal controls. Spine 1998, 23:751-757.

3. Kohyama K, Saura R, Doita M, Mizuno K: Intervertebral disc cell apoptosis by nitric oxide:biological understanding of intervertebral disc degeneration. Kobe J Med Sci 2000, 46:283-295.

4. Ha K-Y, Koh I-J, Kirpalani PA, Kim Y-Y, Cho Y-K, Khang G-S, Han CW: The expression of hypoxia inducible factor- $1 \alpha$ and apoptosis in herniated discs. Spine 2006, 31:1309-1313.

5. Rannou F, Lee T-S, Zhou R-H, Chin J, Lotz JC, Mayoux-Benhamou M-A, Barbet JP, Chevrot A, Shyy JY-L: Intervertebral disc degen- eration. The role of the mitochondrial pathway in annulus fibrosus cell apoptosis induced by overload. Am J Pathol 2004, 164:915-924.

6. Heyde C-E, Tschoeke SK, Hellmuth M, Hostmann A, Ertel W, Oberholzer A: Trauma induces apoptosis in human thoracolumbar intervertebral discs. BMC Clin Pathol 2006, 6:5.

7. Park J-B, Chang H, Kim K-W: Expression of Fas ligand and apoptosis of disc cells in herniated lumbar disc tissue. Spine 2001, 26:618-621.

8. Wyllie AH, Kerr JFR, Currie AR: Cell death: the significance of apoptosis. Int Rev Cytol 1980, 68:251-307.

9. Williams GT, Smith CA, McCarthy NJ, Grimes EA: Apoptosis: final control point in cell biology. Trends Cell Biol 1992, 2:263-267.

10. Jin Z, El-Deiry WS: Overview of cell death signaling pathways. Cancer Biol Ther 2005, 4:139-163.

11. Green DR, Beere H: Gone but not forgotten. Nature 2000, 405:28-29.

12. Kerr JFR, Wyllie AH, Currie AR: Apoptosis: a basic biological phenomenon with wide-ranging implications in tissue kinetics. Br J Cancer 1972, 26:239-257.

13. Roberts $\mathrm{S}$, Evans $\mathrm{H}$, Trivedi J, Menage J: Histology and pathology of the human intervertebral disc. J Bone Joint Surg Am 2006, 88:10-14.

14. Chen B, Fellenberg J, Wang H, Carstens C, Richter W: Occurrence and regional distribution of apoptosis in scoliotic discs. Spine 2005, 30:519-524.

15. Wiegand UK, Corbach S, Prescott AR, Savill J, Spruce BA: The trigger to cell death determines the efficiency with which dying cells are cleared by neighbours. Cell Death Differ 2001, 8:746.

16. Savill J: Recognition and phagocytosis of cells undergoing apoptosis. Br Med Bull 1997, 53:491-508.

17. Tsuchiya S, Yamabe M, Yamaguchi Y, Kobayashi Y, Konno T, Tada $\mathrm{K}$ : Establishment and characterization of a human acute monocytic leukemia line (THP-1). Int J Cancer 1980, 26:171-176.

18. Reyes L, Davidson MK, Thomas LC, Davis JK: Effects of Mycoplasma fermentans incognitus on differentiation of THP-1 Cells. Infect Immun 1999, 67:3188-3192.

19. Oda $T$, Maeda $H$ : A new simple fluorometric assay for phagocytosis. J Immunol Meth 1986, 88:175-183.

20. Johnson WEB: The role of the cytosolic protein, stathmin, in leukaemic cell cycle progression and differentiation [doctoral thesis, Birmingham University, UK]. 1997.

21. Devitt A, Gregory CD: Measurement of apoptotic cell clearance in vitro. In Apoptosis Methods and Protoco/s Edited by: Brady HJM. Totowa: Humana Press Inc; 2004:207-221.

22. Shui Y-B, Sasaki H, Pan J-H, Kojima M, Yamada Y, Hirai K-I, Takahashia N, Sasaki K: Morphological observation on cell death and phagocytosis induced by ultraviolet irradiation in a cultured human lens epithelial cell line. Exp Eye Res 2000, 271:609-618.

23. Fadok VA: Clearance: the last and often forgotten stage of apoptosis. J Mammary Gland Biol Neoplasia 1999, 4:203-211.

24. Bursch W: The autophagosomal-lysosomal compartment in programmed cell death. Cell Death Differ 2001, 8:569-581.

25. Ren Y, Savill J: Apoptosis: the importance of being eaten. Cell Death Differ 1998, 5:563-568.

26. Johnson WEB, Roberts S: 'Rumours of my death may have been grossly exaggerated': a brief review of cell death in human intervertebral disc disease and implications for cell transplantation therapy. Biochem Soc Trans 2007, 35:680-682.

27. Urban JPG, Roberts S: Cells of the intervertebral disc: making the best of a bad environment. The Biochemist 2003, 25:15-17.

28. Castillo ECG, Kouri JB: A new role for chondrocytes as non-professional phagocytes. An in vitro study. Micro Res Tech 2004, 64:269-278.

29. Doita M, Kanatani T, Harada T, Mizuno K: Immunohistologic study of the ruptured intervertebral disc of the lumbar spine. Spine 1996, 21:235-241.

30. Virri J, Sikk S, Gronblad M, Tolonen J, Seitsalo S, Kankare J, Karaharju E: Concomitant immunocytochemical study of macrophage cells and blood cells in disc herniation tissue. Eur Spine J 1994, 3:336-341.

31. Nerlich AG, Weiler C, Zipperer J, Narozny M, Boos N: Immunolocalization of phagocytic cells in normal and degenerated intervertebral discs. Spine 2002, 27:2484-2490. 
32. Pugh DI, Etherington DJ, Silver IA: Enzyme histochemistry of the response by naive and sensitised macrophages to phagocytosis of collagen and latex particles. Z Mikrosk anat Forsche 1985, 99:14-24.

33. Roach HI, Aigner T, Kouri JB: Chondroptosis: a variant of apoptotic death. Apoptosis 2004, 9:265-277.

34. Heinemann DEH, Lohmann C, Alves F, Engel I, Koster G: Human osteoblast-like cells phagocytose metal particles and express the macrphage marker CD68 in vitro. J Bone Jt Surgery $(\mathrm{Br})$ 2000, 82:283-289.

35. Trout JJ, Buckwalter JA, Moore KC: Ultrastructure of the human intervertebral disc: II. Cells of the nucleus pulposus. Anat Rec 1982, 204:307-314.

36. Boos N, Weissbach S, Rohrbach H, Weiler C, Spratt KF, Nerlich AG: Classification of age-related changes in lumbar intervertebral discs. Spine 2002, 27:2631-2644.

37. Roberts S, Evans EH, Kletsas D, Jaffray DC, Eisenstein SM: Senescence in human intervertebral discs. Eur Spine J 2006, 15:S312-S316.

38. Pratsinis H, Kletsas D: PDGF, bFGF and IGF-I stimulate the proliferation of intervertebral disc cells in vitro via the activation of the ERK and Akt signaling pathways. Eur Spine J 2007, 16:1858-1866.

39. Le Maitre C, Freemont AJ, Hoyland JA: Accelerated cellular senescence in degenerate intervertebral discs: a possible role in the pathogenesis of intervertebral disc degeneration. Arthritis Res Ther 2007, 9:R45. 